\title{
Abordagem Deep Learning para Classificação de Lesões Mamárias
}

\author{
Roberto M. P. Pereira ${ }^{1}$, Caio E.F. Matos ${ }^{1}$, João O.B. Diniz ${ }^{1}$, \\ Geraldo Braz Junior ${ }^{1}$, João D. S. De Almeida, Aristófanes Corrêa Silva ${ }^{1}$, \\ Anselmo Cardoso de Paiva ${ }^{1}$
}

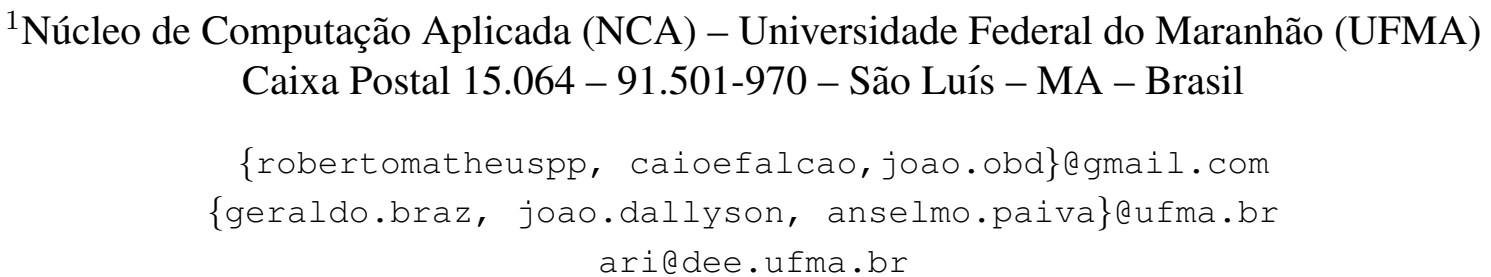

\begin{abstract}
Female breast cancer is a major cause of death in western countries. Several computer aid techniques have been developed seeking to help radiologists in task of detection and diagnosis of breast abnormalities. Recently, deep learning techniques have shown good results in the image classification issues. In this paper, we present a metodology to distinguish mass and normal tissue extracted from mammograms using deep convolutional neural networks.
\end{abstract}

Resumo. O câncer de mama é uma das principais causas de morte de mulheres ocidentais. Várias técnicas têm sido desenvolvidas para auxiliar radiologistas na tarefa de detecção e diagnóstico de lesões mamárias. Recentemente, a técnica de deep learning tem apresentado resultados eficientes na classificação de imagens. Este artigo propõe uma metodologia para classificar tecidos de mamografias em massa e não-massa com o uso de redes neurais convolucionais profundas.

\section{Introdução}

O câncer de mama se manifesta de diferentes maneiras, sendo uma delas caracterizada pelo crescimento de forma anormal de massas. Uma massa é um aglomerado de células que se unem de forma mais densa em relação ao tecido que a envolve. Este aglomerado pode ser causado por câncer de mama, assim como também por condições benignas. Algumas características das massas são determinantes para estabelecer suas probabilidades de malignidade, como por exemplo tamanho, forma e disposição de suas margens.

Medidas de prevenção, como o auto exame ou a utilização de exames de mamografias e ultrassom de rastreamento têm contribuindo para a redução dos índices. A detecção de massas (não se limitando a apenas estas) suspeitas nesses exames é o primeiro passo para o tratamento eficaz de câncer.

Este trabalho tem como objetivo apresentar uma nova metodologia de classificação de regiões extraídas de mamografias em massa e não massa usando técnicas de deep learning. Nessas técnicas, as características são extraídas automaticamente de acordo com a base de treinamento. A contribuição consiste em propôr um método eficiente, de alta sensibilidade e baixa taxa de falso positivos através de deep learning que permite que características diversas sejam extraídas da própria imagem gerando um número maior de peculiaridades relevantes durante a classificação. 


\section{Metodologia}

A metodologia proposta é apresentada na Figura 1, e possui quatro etapas: aquisição, aplicação de filtros, treinamento e resultados.

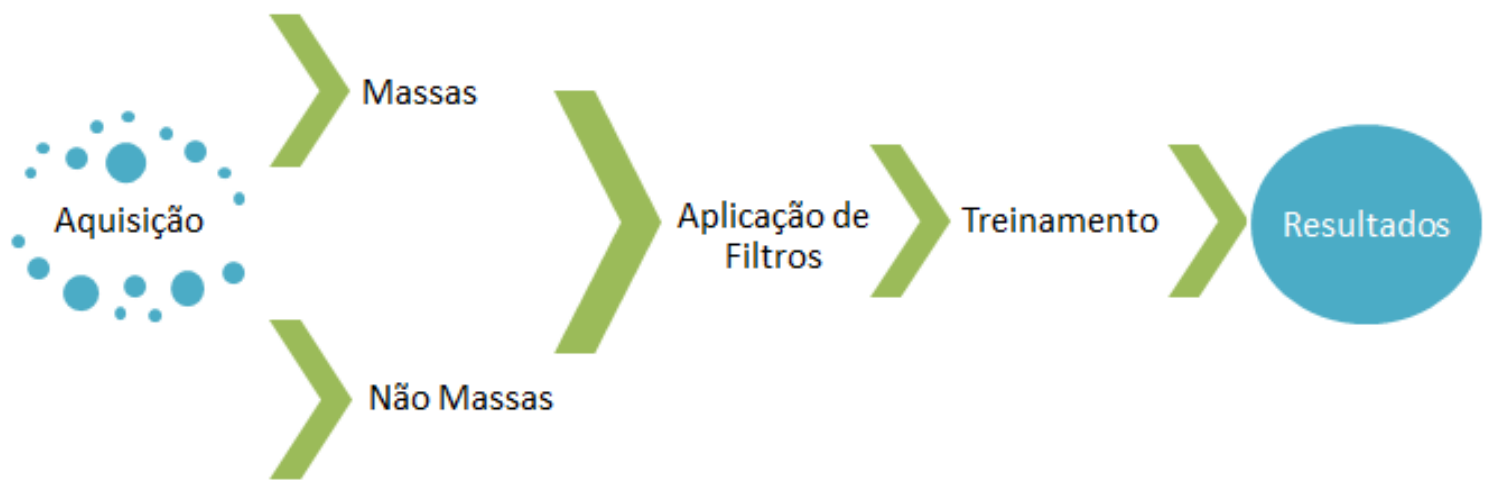

Figura 1. Fluxograma básico da metodologia proposta

\subsection{Aquisição}

A etapa de aquisição foi dedicada à obtenção das imagens mamográficas. Utilizou-se a base pública, de mamografias digitalizadas, Digital Database for Screening Mammography (DDSM) [Heath et al. 1998], disponível na internet.

A seleção das amostras foi realizada com base nas marcações realizadas por especialistas para o caso de massas. Regiões normais foram aleatoriamente retiradas de mamografias que continham massas. O tamanho dos bounding boxes para estas foi definido com base no tamanho médio das regiões que continham massa. Para este trabalho foram utilizados 6.181 regiões, sendo 1.156 diagnosticadas como massas e 5.025 não massas.

\subsection{Aplicação de Filtros}

Com o intuito de verificar o comportamento do resultado das demais etapas, algumas técnicas de preprocessamento foram aplicados nas imagens. Foram testados realce logaritmo e equalização do histograma. Ainda foram utilizadas nos testes as regiões sem nenhum tipo de pre-processamento.

\subsection{Treinamento}

Neste trabalho, foram utilizadas duas arquiteturas de Redes Neurais de Convolução, a AlexNet [Krizhevsky et al. 2012] e a GoogLeNet [Szegedy et al. 2015]. Ambas implementam a ideia de deep learning e mostram resultados promissores na tarefa de classificação de diferentes classes de imagens

Ao todo, cada rede neural foi treinada com 3 instâncias da base de dados original. O primeiro grupo, representa a base de dados aplicando-se o realce logaritmo sobre todas as imagens. O segundo grupo, constitui a base resultante da equalização de histograma das imagens originais e por último, temos a base de dados natural, ou seja, sem a aplicação de nenhum filtro. 
No processo de treinamento, alguns parâmetros das redes AlexNet e GoogLeNet foram modificados, contudo, a arquitetura física das mesmas é mantida durante todos os treinos executados. Os parâmetros alterados foram o número de interações que foi fixado em 90 para ambas as redes, a taxa de aprendizagem foi inicializada em 0,01 e reduzida a cada 30 interações a uma taxa gama de 0,1 , por fim, temos que ambos as redes foram treinadas utilizando o método de gradiente descendente estocástico.

Durante o treino, foram reservados $25 \%$ das imagens da base de dados para teste, $50 \%$ para treino e os $25 \%$ restantes para a validação do treino. Todos as separações treino/teste/validação foram realizadas de maneira aleatória.

\subsection{Validação dos Resultados}

Os resultados são analisados com base nas métricas de Especificidade (Es), Sensibilidade $(S e)$ e Acurácia $(A c)$. A especificidade mostra quão bem o método é em reconhecer tecidos que não possuem massa e é definida por $E s=\frac{V N}{V N+F P}$.

A sensibilidade é definida por $S e=\frac{V P}{V P+F N}$ e mostra a relação de acurácia do método para acerto em massas. Finalmente, a acurácia demonstra a relação global de acerto, definida por $A c=\frac{V P+V N}{V P+V N+F P+F N}$.

\section{Resultados Parciais}

Os experimentos foram conduzidos de acordo com a metodologia apresentada na Seção 2. Os resultados dos testes, obtidos pelas duas arquiteturas de redes neurais são apresentados nas Tabelas 1 e 2 para a AlexNet e a GoogLeNet, respectivamente.

Os melhores resultados foram obtidos utilizando-se a rede neural AlexNet (Tabela 1) sem a aplicação de filtros sobre a base de dados. As transformações de contraste, em todos os testes realizados, apresentaram resultados inferiores à utilização das imagens originais para o teste com a AlexNet.

A estrutura GoogLeNet, contudo mostrou melhoria tanto em relação a acurácia quanto em especificidade quando aplicou-se a equalização de histograma (Tabela 2). Nesse caso, a especificidade é superior aos outros valores obtidos pelas outra arquiteturas, contudo, os valores de sensibilidade e acurácia ainda são inferiores aos obtidos pela AlexNet.

Tabela 1. Resultados encontrados quando os tecidos foram treinados e classificados utilizando-se a AlexNet

\begin{tabular}{|l|c|c|c|}
\cline { 2 - 4 } \multicolumn{1}{c|}{} & Sensibilidade & Especificidade & Acurácia \\
\hline Histograma Equalizado & 0,7024 & 0,965 & 0,9159 \\
\hline Base de Dagarítmico & 0,6886 & 0,9586 & 0,9081 \\
\hline
\end{tabular}

Através dos resultados podemos observar que as redes tiveram alta taxa de reconhecimento de regiões normais, indicando um melhor aprendizado das amostras que tinham a maior prevalência na base. Regiões normais são também regiões de fundo bem definida. Regiões de massa, embora mais homogêneas possuem larga variação de forma e textura. As arquiteturas representaram um menor poder de aprendizado de massas, embora ainda significativo. 
Tabela 2. Resultados encontrados quando os tecidos foram treinados e classificados utilizando-se a GoogLeNet

\begin{tabular}{|l|c|c|c|}
\cline { 2 - 4 } \multicolumn{1}{c|}{} & Sensibilidade & Especificidade & Acurácia \\
\hline Histograma Equalizado & 0,5986 & 0,9705 & 0,901 \\
\hline Realce Logarítmico & 0,2845 & 0,9299 & 0,7427 \\
\hline Base de Dados Original & 0.6021 & 0,9315 & 0,8699 \\
\hline
\end{tabular}

\section{Conclusão e Próximos Passos}

Neste trabalho foi apresentada uma aplicação de $C N N$, para a classificação de tecidos de mamografia, em massa e normal.

As mamografias são imagens de pouca informação de textura. As massas presentes nesse tipo de imagem apresentam em sua maioria grande semelhança com regiões próximas, tornando o trabalho de detecção dependente do ajuste de inúmeros parâmetros. Pensando nisso, este trabalho apresentou a classificação de massas e regiões normais usando arquiteturas deep learning como uma alternativa da aplicação de técnicas de grande parametrização. Arquiteturas deste tipo são recentes e sua grande vantagem consiste no autoaprendizado de características e na robustez de classificação.

A intenção é a construção de uma abordagem altamente adaptável e automatizada na detecção de massas em mamografias. Os resultados apresentados, utilizando-se as arquiteturas AlexNet e GoogLeNet foram promissores embora sejam necessários ajustes para o correto aprendizado de regiões de massa.

Um dos problemas encontrados até o momento consiste no desbalanceamento da base que pretendemos corrigir utilizando a técnica SMOTE [Chawla et al. 2002]. Ainda como continuação, pretendemos ajustar corretamente o erro retropropagado para ajuste de sensibilidade e a construção das camadas de convolução de acordo com textura de baixa informação, como no caso de regiões de mamografia.

\section{Agradecimento}

Os autores desejam agradecer o apoio financeiro concedido pela FAPEMA e CNPq para a execução desta pesquisa.

\section{Referências}

Chawla, N. V., Bowyer, K. W., Hall, L. O., and Kegelmeyer, W. P. (2002). Smote: synthetic minority over-sampling technique. Journal of artificial intelligence research, pages 321-357.

Heath, M., Bowyer, K., Kopans, D., Kegelmeyer Jr, P., Moore, R., Chang, K., and Munishkumaran, S. (1998). Current status of the digital database for screening mammography. In Digital mammography, pages 457-460. Springer.

Krizhevsky, A., Sutskever, I., and Hinton, G. E. (2012). Imagenet classification with deep convolutional neural networks. In Advances in neural information processing systems, pages 1097-1105.

Szegedy, C., Liu, W., Jia, Y., Sermanet, P., Reed, S., Anguelov, D., Erhan, D., Vanhoucke, V., and Rabinovich, A. (2015). Going deeper with convolutions. In Proceedings of the IEEE Conference on Computer Vision and Pattern Recognition, pages 1-9. 